\title{
Magellanic Cloud Planetary Nebulae
}

\author{
Richard A. Shaw ${ }^{1}$ \\ ${ }^{1}$ National Optical Astronomy Observatory, Tucson, AZ 85719, USA \\ email: shaw@noao.edu
}

\begin{abstract}
Over the past 15 years the growth of detailed information about Planetary Nebulae in the Large and Small Magellanic Clouds (MCPN) has been explosive, to the point where these galactic laboratories are the preferred context for furthering and refining our understanding of this late phase of stellar evolution. Deep, photometrically uniform surveys have pushed the population of confirmed MCPN above 800, good-quality optical spectra exist for the bulk of these nebulae, infrared and satellite UV spectra of a few dozen PNe are archived or will become available soon, and high-resolution $(H S T)$ images have been archived for over 150 nebulae. In this paper I review the accumulated knowledge of MCPN: including physical and chemical properties of the nebulae and their relation to morphological characteristics; central star properties and what they tell us about the progenitor population; and what new insight has been gained from MCPN about the evolution of the combined PN+central star systems.
\end{abstract}

Keywords. planetary nebulae: general, Magellanic Clouds, stars: evolution

\section{Introduction}

Planetary Nebulae in the Large and Small Magellanic Clouds (MCPN) have been studied for nearly half a century. The initial emphasis was to understand the stellar populations and structure of the Clouds themselves, but by the 1960s studies of the physical properties of the nebulae and the evolution of their central stars was well underway (see Westerlund 1968, and references therein). Interest in MCPNe has grown significantly over time and a good deal of progress in our understanding of planetary nebulae (PNe) in this context has been made over the past four decades, as summarized in reviews presented in this series of Symposia (Webster 1978; Jacoby 1983; Barlow 1989; Dopita 1993, 1997; Stanghellini 2003). The advantages of studying PNe in the Clouds have been described by many. Briefly, PNe within each Cloud all lie at a common distance that is known to $\sim 10 \%$ accuracy; the old stellar population within the Clouds is sufficiently large that they contain hundreds of PNe, and they are sufficiently close that high quality spectra can be obtained with modern observing facilities; the foreground extinction is low so that there is little selection bias; and the average metallicity of each Cloud is different (and lower than that of the Galaxy), making comparative studies of metallicity effects illuminating.

A few important advances in the past few years will change the landscape of MCPN research: large numbers of new $\mathrm{PNe}$ are being discovered in deep, wide, photometrically uniform surveys that, so far, have pushed the population of PNe above 350 in the LMC, and to about 135 in the SMC. Also, large numbers of MCPN have been imaged at high resolution with $H S T$, so that critical information on the nebular morphologies and sizes, and on the stellar magnitudes (or limits thereto) is now available for $\sim 150 \mathrm{PNe}$. Goodquality optical spectra exist for the bulk of these nebulae, infrared spectra from Spitzer have been obtained for dozens of MCPNe (Bernard-Salas 2006; Stanghellini et al. 2006), and satellite UV spectra of several more PNe have been published (Lee, Stanghellini \& Shaw 2006). In this paper I review the bounds of our knowledge of MCPN, with special 
emphasis on recent results: including physical and chemical properties of the nebulae, and their relation to morphological characteristics; central star properties and what they tell us about the progenitor population; and what new insight has been gained through MCPN into the evolution of the combined PN+central star systems, and the implications for understanding the physical origin of the PN luminosity function (PNLF).

\section{Nebulae}

What we know about MCPN is strongly shaped by the available discovery surveys. The early, pioneering surveys for PNe are characterized by a variety of search techniques, spatial coverage, photometric depth, source confusion, and confirmation criteria (see the reviews by Jacoby 2003; Parker 2006). Source samples drawn from heterogeneous surveys (that are often restricted to the brightest PNe) limit the extent to which generalizations can be made about global properties of MCPN, or of inferences that can be drawn about properties of the host galaxies based on PNe. Specifically, the identification of PNe in the central portion of the SMC is complete for the first $\sim 7$ mag of the [O III] luminosity function (Jacoby \& De Marco 2002), but is far less complete elsewhere. HST images exist for roughly $70 \%$ of all known SMC PNe, but only for about $25 \%$ of those in the Jacoby \& De Marco (2002) sample. The completeness in the LMC is at the moment far worse: absolute [O III] fluxes have been published for only about 150 of the $\sim 350$ known nebulae, of which HST has imaged 114 to date. The next generation of deep, wide-field, and uniform surveys (Smith 1999; Reid \& Parker 2006), when published in their entirety, should improve the completeness considerably. Reid \& Parker (2006), in particular, discovered 73 new PNe in a subset of their survey, and will soon add more than 350 within the central 25 square degrees of the LMC (Reid, private communication). A summary of general properties of MCPN, mostly drawn from the HST sample described by Shaw et al. (2006), is given in Table 1. Broadly speaking, most of the nebulae are bright and small and suffer little foreground extinction. The other properties will be discussed in more detail in the following sections.

Table 1. General Properties of MCPN

\begin{tabular}{lrccccc}
\hline Galaxy & $\begin{array}{r}\text { Number } \\
\text { Known }\end{array}$ & $\begin{array}{c}\text { Bipolar } \\
(\%)\end{array}$ & $\begin{array}{c}H S T \\
\text { Images }\end{array}$ & $\begin{array}{c}\langle\text { Radius } \\
(\mathrm{pc})\end{array}$ & $\langle c\rangle$ & $\begin{array}{c}\left\langle\mathrm{M}_{C S}\right\rangle \\
\left(\mathrm{M}_{\odot}\right)\end{array}$ \\
\hline LMC & 700 & 51 & 114 & 0.10 & 0.19 & 0.65 \\
SMC & 135 & 30 & 35 & 0.08 & 0.10 & 0.63 \\
\hline
\end{tabular}

\subsection{Morphology}

Shaw et al. (2001, 2006) and Stanghellini et al. (2002a) classified the morphologies of MCPN into six broad categories: Round, Elliptical, Bipolar, Bipolar-core, Quadrupolar, and Point-symmetric. These classifications, while generally correct, were not informed by detailed maps of the velocity structure of the nebula. Thus, a few PNe classified as E or R could be B, but viewed nearly pole-on. Nevertheless, studying the correlation of morphological type with various physical properties is illuminating. The fraction of $\mathrm{PNe}$ with axi-symmetric features (including $\mathrm{B}, \mathrm{BC}$, and $\mathrm{Q}$ types) is distinctly larger in the LMC than in the SMC or the Galaxy $(26 \%)$, which may relate to the metallicity of the progenitor stars (Stanghellini et al. 2000) or to the star-formation history of the host galaxies. Reid \& Parker (2006) found faint haloes associated with approximately $60 \%$ of LMC PNe. These haloes, which are fainter than the main shell by a factor of 100 to 1000 , 

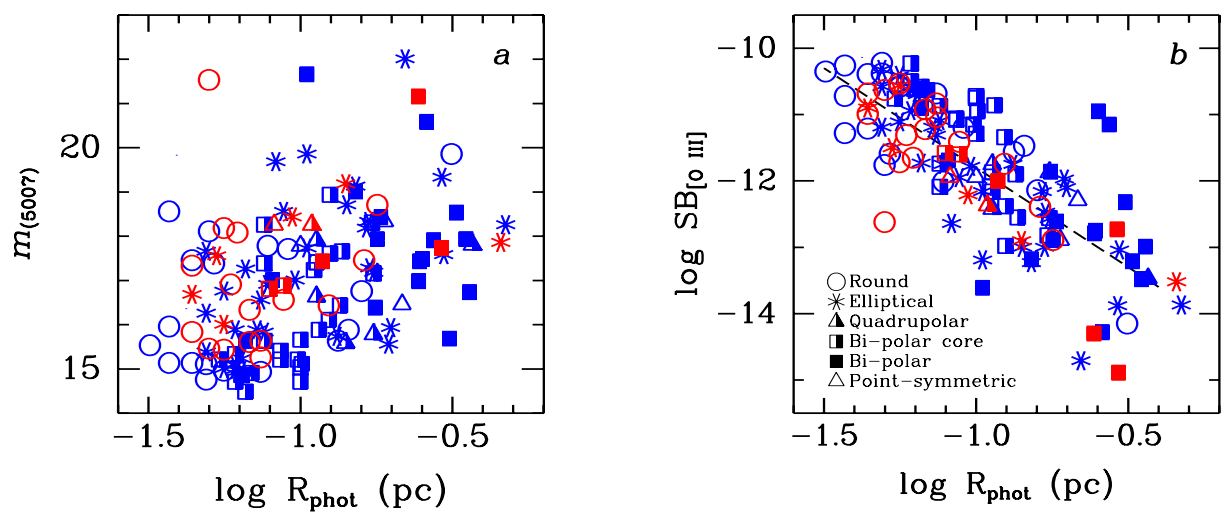

Figure 1. Plot a, at left: Variation of the flux in [O III $] \lambda 5007$, expressed in magnitudes with photometric radius for the LMC (blue) and SMC (red) nebulae. Plot $\mathbf{b}$, at right: Decline of extinction-corrected average surface brightness in [O III $\lambda 5007$ with $\mathrm{R}_{\text {phot }}$ is consistent with an $\mathrm{R}^{-3}$ power law (dotted line). Symbols indicate morphological type, as shown in the legend.

may be the analogs of the faint AGB haloes found by Corradi et al. (2003) for Galactic $\mathrm{PNe}$, with roughly the same frequency.

Figure $1 a$ shows that the PNLF samples a wide range of nebular properties, including the photometric radius $\left(\mathrm{R}_{p h o t}\right)$ and morphological type (and therefore also chemical abundances; see below) for all magnitude bins except for the brightest two, where bipolar $\mathrm{PNe}$ are distinctly under-represented. This property may contribute to the robustness of the PNLF over a wide range of galaxy type and average metallicity. The decline in average nebular surface brightness with photometric radius, described by Shaw et al. (2006) for an ensemble of MCPNe, is reproduced in Figure $1 b$. The decline can be characterized by a power-law with an index of -3 . Frew (2006) has employed this relation as a distance indicator for Galactic PNe, but instead using $\mathrm{H} \alpha$ emission which is less affected by variations in nebular excitation. In [O III], however, MCPNe with log radii larger than about -0.7 , the majority of which are bipolar, show a huge dispersion from the trend. This effect might be expected for optically thick nebulae where the CS evolves quickly to low luminosity, and therefore cannot fully ionize the surrounding nebula. In this case the measured radius is that of the ionization front rather than the edge of the main shell, and it would increase only in those cases where the CS produces sufficient ionizing photons. Such a circumstance might be expected for the most massive CSs that are predicted to produce the most massive $\mathrm{PNe}$; that the bulk of these nebulae are bipolar is consistent with what is known for Galactic PNe. The optical depth also depends upon the gas density, which should change substantially as the nebula evolves. Figure 2 (Shaw et al. 2006) shows the decline of average nebular density with radius, where again the (mostly bipolar) nebulae with log radii larger than -0.7 have much higher density than would be expected from the trend line. This is another indication that many of these nebulae are still optically thick at large radius.

\subsection{Abundances}

Large spectroscopic studies of MCPN (e.g., Leisy \& Dennefeld 1996; Stasinska, Richer \& Mc Call 1998) have enabled a close examination of nebular abundance variations with morphology. As shown in Figure 3, bipolar nebulae are typified by extreme ratios of $\mathrm{F}([\mathrm{N} \mathrm{II}]) / \mathrm{F}(\mathrm{H} \alpha)$ and are enhanced in N (Shaw et al. 2006). As in the Galaxy, bipolar MCPN are preferentially, though perhaps not exclusively, of Type 1 (Torres-Peimbert \& 


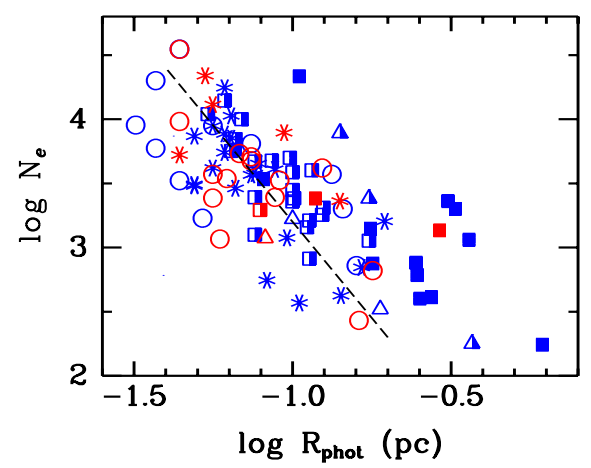

Figure 2. Decline of nebular density with photometric radius for the LMC (blue) and SMC (red) nebulae. Symbols indicate morphological type, as shown in Fig. 1. Dashed line shows $N_{e} \propto R^{-3}$.
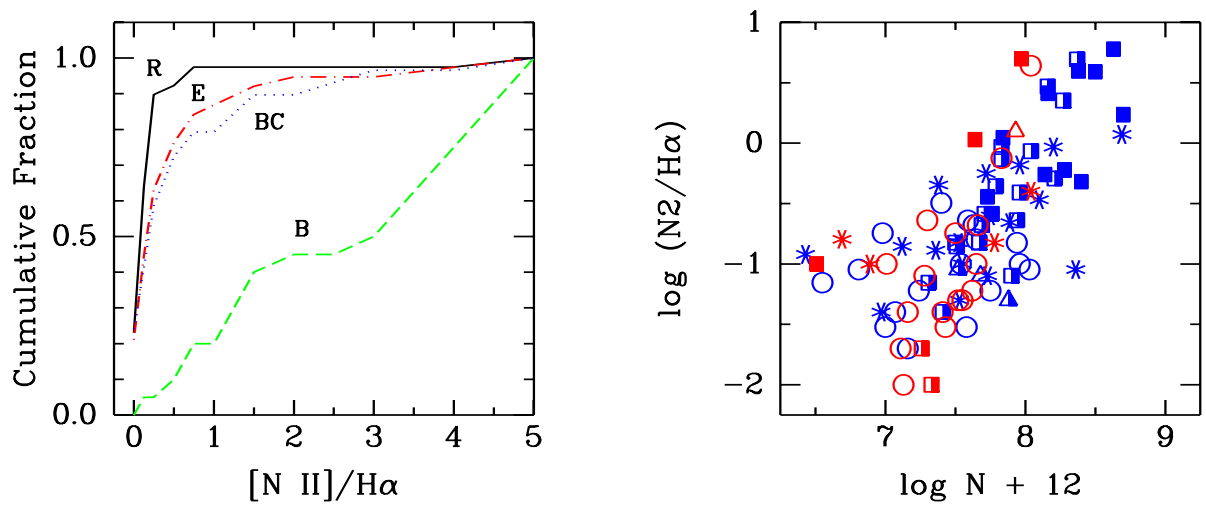

Figure 3. Plot a, at left: Cumulative fraction of MCPN with values of $\mathrm{F}([\mathrm{N} \mathrm{II}]) / \mathrm{F}(\mathrm{H} \alpha)$, where the [N II] flux is the sum of the fluxes in the emission lines $\lambda 6548$ and $\lambda 6583$. Morphological types are indicated by different curves. Plot $\mathbf{b}$, at right: $\mathrm{F}([\mathrm{N} \mathrm{II}]) / \mathrm{F}(\mathrm{H} \alpha)$ vs. $\mathrm{N}$ for the $\mathrm{LMC}$ (blue) and SMC (red) nebulae, showing that high values of $\mathrm{N} 2 / \mathrm{H} \alpha$ are a good proxy for elevated $\mathrm{N}$ abundance. Symbols indicate morphological type, as in Fig. 1.

Peimbert 1997), showing one or (often) both of elevated He abundance and high N/O. Stanghellini et al. (2000) showed preliminary correlations of LMC PNe abundance patterns with morphology, including the familiar inverse relationship between $\mathrm{C} / \mathrm{O}$ and N/O. These results are updated in Figure 4 with the availability of many more morphological classifications, including PNe in the SMC. Note the tendency of bipolars to show the greatest depletions of $\mathrm{C}$ and enhancements of $\mathrm{N}$, which is attributed to hot-bottom burning after the final episode of dredge-up from the AGB core (Herwig 2005). Stanghellini et al. (2006) noted that gas-phase abundances of these elements appear to be related to the presence of IR silicate and PAH spectral features. It is also important to note that the spread of abundances in $\mathrm{CNO}$ and $\mathrm{He}$ is far larger than the difference between the average abundances of LMC and SMC PNe in these elements.

\section{Central Stars}

Central stars (CSs) have been studied, albeit indirectly, in MCPN for nearly two decades using a variety of techniques (see Table 2). With the advent of HST 

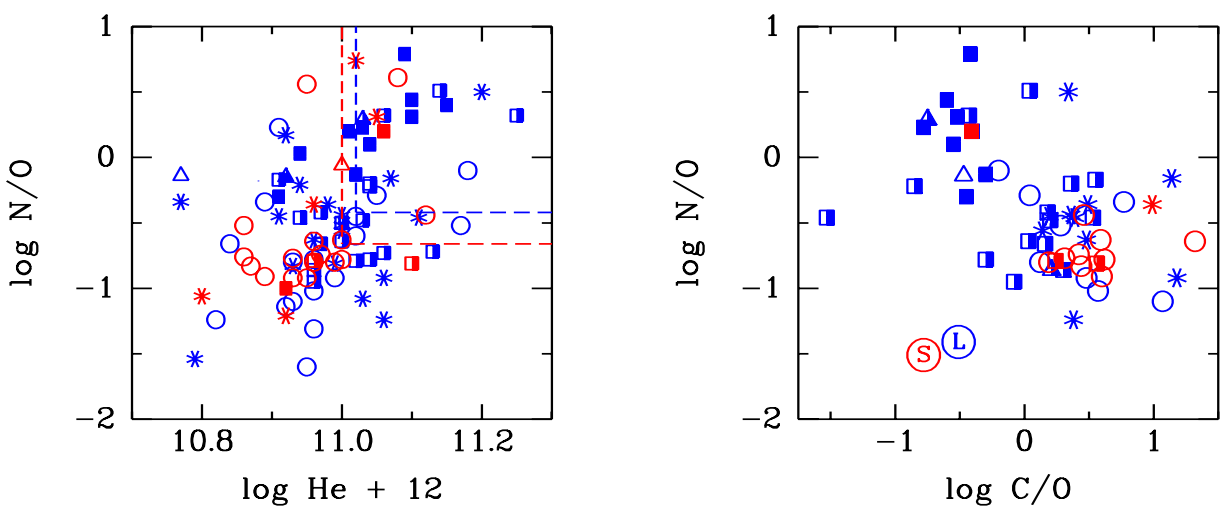

Figure 4. Plot a, at left: N/O vs. He for the LMC (blue) and SMC (red) nebulae. Regions of Type 1 PNe as revised by Torres-Peimbert \& Peimbert (1997) are indicated (dashed lines). Plot b, at right: $\mathrm{C} / \mathrm{O}$ vs. N/O for the LMC (blue) and SMC (red) nebulae. Average abundances for $\mathrm{H}$ II regions is indicated. Symbols indicate morphological type, as in Fig. 1.

Table 2. Studies of CS Temperature \& Luminosity

\begin{tabular}{lll}
\hline Reference & Abbr. & Technique \\
\hline Henry, Liebert \& Boroson (1989) & HLB & Stoy, L $(\mathrm{H} \beta)$ \\
Dopita \& Meatheringham (1991a,b) & DM & Empirical: excitation class, L(H $\beta)$ \\
Kaler \& Jacoby (1990, 1991) & KJ & H/He crossover \\
Herald \& Bianchi (2004) & HB & Model atmosphere fit \\
Villaver, Stanghellini \& Shaw (2003, 2004, 2006) & VSS & Zanstra, Bolometric correction \\
\hline
\end{tabular}

observations, CSs can now be resolved from the nebula and background stars, and quite accurate temperatures and luminosities can be derived (Herald \& Bianchi 2004; Villaver, Stanghellini \& Shaw 2003, 2004, 2006). Accurate CS masses are needed to understand whether predicted correlations of nebular and CS properties are realized in practice, and also to derive the mass distribution for comparison with that for PNe in the Galaxy and with that for white dwarfs. For the latter purpose it is important to determine individual CS masses to within a few hundredths of a solar mass, which requires high accuracy in the parameters used to derive the masses. Perhaps the most widely applied technique is to compare the CS luminosity and temperature to the published stellar evolution tracks. In order to determine masses to within $0.03 \mathrm{M}_{\odot}$, it is necessary to determine $\log \mathrm{L}$ to within $\sim 0.1$ (at least on the constant-luminosity portion of the tracks), and $\log \mathrm{T}$ to within $\sim 0.05$. Because of the significant variation of evolution tracks with progenitor metallicity, and the large range of metallicity in the $\mathrm{PN}$ population in each Cloud, it is also critical to determine the metallicity of the progenitor to within $\sim 0$. dex. But as Figure 5 shows, the agreement in $\mathrm{L}$ and $\mathrm{T}$ among the authors cited in Table 2 is generally quite poor. The two most accurate techniques for MCPN, when the CS is unambiguously detected, are model atmosphere fitting (when high resolution, high $\mathrm{S} / \mathrm{N}$ spectra in the UV and optical are available), and the Zanstra temperatures with L determined from a bolometric correction. Note that previously published correlations between CS masses as derived from other techniques, and various nebular properties such as extinction and nebular mass are probably not reliable.

The mass distribution of the MCPN central stars from VSS is shown in Figure 6. The mean mass is roughly the same for each Cloud, near $0.65 \mathrm{M}_{\odot}$. The means are slightly 

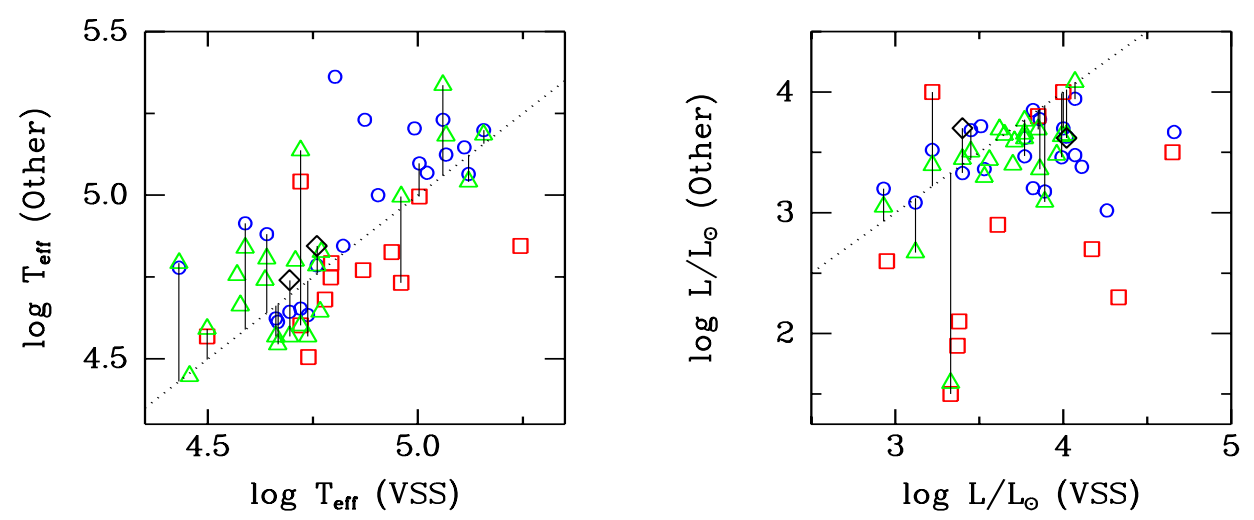

Figure 5. Comparison of derived temperatures (left) and luminosities (right) for CSs in the MC from HLB (squares), KJ (triangles), DM (circles), and HB (diamonds), to those of VSS. The 1:1 line (dotted) is shown for reference; determinations for stars by more than two authors are connected with a thin, vertical line. The desired accuracy is roughly the size of the symbols.

higher than that for Galactic PNe (Stanghellini et al. 2002b) and white dwarfs (Liebert, Bergeron \& Holberg 2005), which if taken at face value could be evidence of higher core masses being produced for a given progenitor mass in an environment with lower average metallicity (Herwig 2005). There is also a suggestion, albeit with low statistical significance, that the distributions are different. If true, it could be explained by elevated star formation in the LMC as recently as $10^{9}$ years ago, such that progenitor stars with masses a bit larger than $2 \mathrm{M}_{\odot}$ are producing excess CSs near $0.7 \mathrm{M}_{\odot}$.

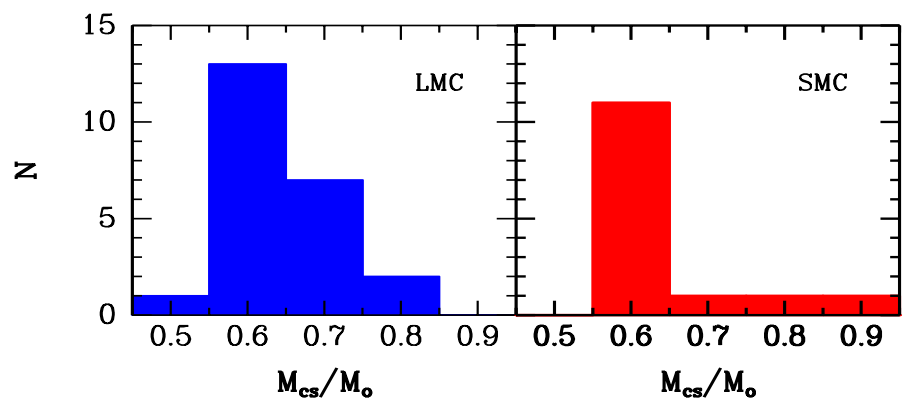

Figure 6. Distribution of CS masses for the LMC (left) adapted from Villaver, Stanghellini \& Shaw $(2003,2006)$, and for the SMC (right) from Villaver, Stanghellini \& Shaw (2004).

VSS did not find any correlation of the CS masses with nebular morphology, as has been reported for Galactic PNe. Neither is there any correlation between extinction (as measured from the nebular Balmer decrement) and morphological type (Stanghellini et al. 2002a), nor with CS mass. It is possible, however, that with more data the expected correlation of high CS mass and bipolar morphology will become apparent. VSS were not able to detect CSs embedded in very bright nebulosity, and often the nebulae with bright continua were bipolar. Also, the sensitivity limits of the VSS sample meant that few stars less luminous than $\log \mathrm{L} / \mathrm{L}_{\odot} \leqslant 2.8$ were represented, which is at the upper end of where the most massive CSs would be found, given their swift evolution through the $\mathrm{PN}$ portion of the H-R diagram. Interestingly, the magnitudes of the CSs in the VSS sample exclude for all but a very few objects the possibility of a binary companion that 
is comparably bright in the STIS UV/Visual/near-IR bandpass. White dwarf and brown dwarf companions cannot be excluded, however.

\section{Co-Evolution of the Nebula and Central Star}

One of the most important advances in the past few years has been the development of hydrodynamic models that accurately track the evolution of the nebular gas in response to evolving temperature, luminosity, and wind momentum of the CS. Models published by Villaver, Manchado \& Garcia-Segura (2002) and Perinotto et al. (2004) show that important morphological properties of PNe, such as double shells and faint, outer haloes can be understood in terms of a complex interaction of shock waves and an ionization front that advances through the nebula. Remarkably, these models also show that the most massive central stars, which also eject the greatest amount of mass during the prior AGB phase, will not fully ionize their surrounding nebulae and will remain optically thick during the entire PN phase of evolution. In addition, the velocity structure of the gas is more strongly influenced by internal shocks and the dynamics of the ionization front than by simple expansion of the PN main shell. The implications of these results are profound, and at the least will require a reinterpretation of published nebular dynamical ages and of expansion parallaxes in the context of hydrodynamic models.

\section{Summary and Future Directions}

Planetary Nebulae in the Magellanic Clouds present a unique opportunity to understand the late evolutionary stages of low- to intermediate-mass stars in a way that is difficult or impossible to realize with Galactic PN samples. Recent ground- and spacebased surveys of MCPNe have already added rich detail to the understanding of PNe developed from Galactic samples, and have raised new and challenging questions. New surveys, using more powerful instrumentation and techniques, make it possible to discover and study all of the hundreds of PNe in each Cloud, and to address such fundamental questions as the fraction of stars that produce observable PNe, the historical rate of mass loss on the AGB, the fraction of CSs that have binary companions, the factors that govern the shaping and evolution of the PN shells, and the nucleosynthetic processes that operate within AGB stars.

\section{Acknowledgements}

Support for this presentation is gratefully acknowledged from NASA through grants GO-09077, GO-09120, and GO-10251 from Space Telescope Science Institute, which is operated by the Association of Universities for Research in Astronomy, Inc., under NASA contract NAS5-26555.

\section{References}

Barlow, M. J. 1989, in IAU Symp. 131, Planetary Nebulae, ed. S. Torres-Peimbert (Dordrecht: Kluwer), 319

Bernard-Salas, J. 2006, in IAU Symp. 234, Planetary Nebulae in Our Galaxy and Beyond, ed. M.J. Barlow \& R.H. Mendez (Cambridge, UK: Cambridge Univ. Press), these proceedings Corradi, R. L. M., Schönberner, D., Steffen, M., \& Perinotto, M. 2003, MNRAS, 340, 417

Dopita, M. A. \& Meatheringham, S. J. 1991a, ApJ, 367, 115

Dopita, M. A. \& Meatheringham, S. J. 1991b, ApJ, 377, 480

Dopita, M. A. 1993, in IAU Symp. 155, Planetary Nebulae, ed. R. Weinberger \& A. Acker (Dordrecht: Kluwer), 433 
Dopita, M. A. 1997, in IAU Symp. 180, Planetary Nebulae, ed. H. J. Habing \& H. Lamers (Dordrecht: Kluwer), 417

Frew, D. 2006, in IAU Symp. 234, Planetary Nebulae in Our Galaxy and Beyond, ed. M.J. Barlow \& R.H. Mendez (Cambridge, UK: Cambridge Univ. Press), these proceedings

Henry, R. B. C., Liebert, J., \& Boroson, T. A. 1989, ApJ, 339, 872

Herald, J. E. \& Bianchi, L. 2004, ApJ, 611, 294

Herwig, F. 2005, ARAA, 43, 435

Jacoby, G. H. 1983, in IAU Symp. 103, Planetary Nebulae, ed. D. R. Flower (Dordrecht: Reidel), 427

Jacoby, G. H. \& De Marco, O. 2002, AJ, 123, 269

Jacoby, G. H. 2003, in Planetary Nebulae Beyond the Milky Way, ed. L. Stanghellini, J. Walsh \& N. G. Douglas (Berlin: Springer), 17

Kaler, J. B. \& Jacoby, G. H. 1990, ApJ, 362, 491

Kaler, J. B. \& Jacoby, G. H. 1991, ApJ, 382, 134

Lee, T.-H., Stanghellini, L., \& Shaw, R. A. 2006, in IAU Symp. 234, Planetary Nebulae in Our Galaxy and Beyond, ed. M.J. Barlow \& R.H. Mendez (Cambridge, UK: Cambridge Univ. Press), these proceedings

Liebert, J., Bergeron, P., \& Holberg, J. B. 2005, ApJS, 156, 47

Leisy, P. \& Dennefeld, M. 1996, in IAU Symp. 155, Planetary Nebulae, ed. R. Weinberger \& A. Acker (Dordrecht: Kluwer), 433

Parker, Q. 2006, in IAU Symp. 234, Planetary Nebulae in Our Galaxy and Beyond, ed. M.J. Barlow \& R.H. Mendez (Cambridge, UK: Cambridge Univ. Press), these proceedings

Perinotto, M., Schönberner, D., Steffen, M., \& Calonaci, C. 2004, A\&AA, 414, 993

Reid, W. A. \& Parker, Q. A. 2006, MNRAS, 365, 401

Shaw, R. A., Stanghellini, L., Mutchler, M., Balick, B., \& Blades, J. C. 2001, ApJ, 548, 727

Shaw, R. A., Stanghellini, L., Villaver, E., \& Mutchler, M. 2006, ApJ, in press (astro-ph/0608300)

Smith, R. C. 1999, in IAU Symp. 190, New Views of the Magellanic Clouds, ed. Y.-H. Chu, N.B. Suntzeff, J.E. Hesser \& D.A. Bohlender (San Francisco: ASP), 28

Stanghellini, L., Shaw, R. A., Balick, B., \& Blades, J. C. 2000, ApJ, 534, L167

Stanghellini, L., Shaw, R. A., Mutchler, M., Palen, S., Balick, B., \& Blades, J. C. 2002a, ApJ, 575,178

Stanghellini, L., Villaver, E., Manchado, A., \& Guerrero, M. A. 2002b, ApJ, 576, 285

Stanghellini, L. 2003, in IAU Symp. 209, Planetary Nebulae: Their Evolution and Role in the Universe, ed. S. Kwok, M. Doptia, \& R. Sutherland (San Francisco: ASP), 567

Stanghellini, L., Shaw, R. A., Balick, B., Mutchler, M., Blades, J. C., \& Villaver, E. 2003, ApJ, 596, 997

Stanghellini, L., Garcia-Lario, P., Manchado, A., Vicente-Perea, J., Garcia-Hernandez, A., Shaw, R. A., \& Villaver, E. 2006, in IAU Symp. 234, Planetary Nebulae in Our Galaxy and Beyond, ed. M.J. Barlow \& R.H. Mendez (Cambridge, UK: Cambridge Univ. Press), these proceedings

Stasinska, G., Richer, M. G., \& Mc Call, M. L. 1998, AAp, 336, 667

Torres-Peimbert, S. \& Peimbert, M. 1997, in IAU Symp. 180, Planetary Nebulae, eds. H. J. Habing \& H. Lamers (Dordrecht: Kluwer), 175

Villaver, E., Manchado, A., \& Garcia-Segura, G. 2002, ApJ, 581, 1204

Villaver, E., Stanghellini, L., \& Shaw, R. A. 2003, ApJ, 597, 298

Villaver, E., Stanghellini, L., \& Shaw, R. A. 2004, ApJ, 614, 716

Villaver, E., Stanghellini, L., \& Shaw, R. A. 2006, ApJ, in press (astro-ph/0610079)

Webster, B. 1978, in IAU Symp. 76, Planetary Nebulae, ed. Y. Terzian (Dordrecht: Kluwer), 11

Westerlund, B. 1968, in IAU Symp. 34, Planetary Nebulae, ed. D. E. Osterbrock, \& C. R. O'Dell (Dordrecht: Reidel), 23 